\section{Speech perception and psychophysics: Theory and experiment}

\section{DOMINIC W. MASSARO and JOSEPH M. HARY University of California, Santa Cruz, California}

Macmillan's comments are welcome and give us the opportunity to elaborate on some important points concerning the issue of categorical perception. In this reply, we argue that our interpretation of the Hary and Massaro (1982) results is not paradoxical if the important differences between a theory of perception and the results used to support the theory are respected. Next, we clarify the goals of the Hary and Massaro study and contrast the traditional categorical account against Macmillan's psychophysical account. Athough we conclude, as did Hary and Massaro (1982) and Macmillan (1983), that perception of the pluck/bow continuum is probably continuous and not categorical, our main point is that this hypothesis cannot be adequately tested using the identificationdiscrimination task.

\section{Distinguishing Between Theory and Results}

It is important to distinguish between a theory of perception and the results used to support the theory. As defined by Studdert-Kennedy, Liberman, Harris, and Cooper (1970), categorical perception is "a mode by which stimuli are responded to, and can only be responded to, in absolute terms"' (p. 234). According to this theory, the perceptual system limits the listener to categorical information about certain speech events. If the theory of categorical perception is correct, then discrimination of certain speech sounds should be limited by identification (Liberman, Harris, Hoffman, \& Griffith, 1957). In this case, categorical-perception theory predicts the observable result that two different speech sounds can be discriminated only to the extent that they are identified differently. Thus, the theory predicts that identification performance can predict discrimination performance and results consistent with this prediction are usually taken as evidence for the theory of categorical perception (e.g., Healy \& Repp, 1982; Liberman et al., 1957; Studdert-Kennedy et al., 1970).

One fallacy involved in this research endeavor is that empirical agreement with a theory's predictions does not verify the theory; it only fails to falsify the

This research was supported by a grant from the National Institute of Mental Health (MH-35334) to Dominic W. Massaro. Thanks are extended to Ray Gibbs for helpful comments on an earlier version of this paper and to Neil Macmillan for useful discussions. Requests for reprints should be sent to Dominic W. Massaro, Program in Experimental Psychology, University of California, Santa Cruz, Santa Cruz, California 95064. theory (Popper, 1959). What the advocates of categorical perception failed to demonstrate was that the same results falsified alternative theories. The obvious alternative theory is that of continuous perception in which listener's have continuous, not just categorical, information about a stimulus event. A necessary question of the research should have been: Can continuous perception also predict a direct relationship between identification and discrimination? If it could not, the results would have been highly informative with respect to the issue of categorical versus continuous perception. If continuous perception can also predict such a relationship, however, the results are completely uninformative.

It turns out that continuous perception can also predict a direct relationship between identification and discrimination. Macmillan, Kaplan, and Creelman (1977) expanded on the ideas of Durlach and Braida (1969) and showed that exactly such a direct relationship is possible, even if the underlying information is continuous. The underlying information was defined in terms of Thurstone's theory of comparative judgment and signal detection theory. Given this observation, however, Macmillan et al. (1977) did not question the value of the identification-discrimination task; they simply redefined the two contrasting modes of perception.

Macmillan et al. (1977) defined categorical perception as equivalent discrimination and identification performance (when analyzed in $\mathrm{d}^{\prime}$ values) and continuous perception as significantly better performance in discrimination than in identification. Unfortunately, this definition reduces an important theoretical question to an empirical observation. Although the authors distinguished between two possible empirical results, they provided no insights into the implications of these results for theory. In their reanalysis of the Cutting and Rosner (1974) experiments, a nonspeech continuum (pluck/bow) gave nearly categorical results and a speech continuum (chop/shop) gave continuous results. However, the authors made no attempt to explain these surprising differences and they did not question whether their new analysis was more meaningful than the old. We believe that the differences between the two continua simply reflect the fact that the chop/shop continuum did not have very good instances of the two categories, even at extremes of the continuum. No instance along this continuum was identified as a given category more than $90 \%$ of the time. In general, poor identification performance relative to discrimination will be observed whenever the continuum does not contain good instances of the categories being identified.

What is ironic is that Macmillan et al. did not use their observations to question the original conclu- 
sions of advocates of categorical perception. They simply reduced categorical perception to an empirical result in which the perceived spacing of signals along a dimension is observed to be the same for identification and for discrimination. However, what their paper implied, although they failed to acknowledge it, was that the observed relationship between identification and discrimination was no longer diagnostic of the original theories of categorical and continuous perception.

\section{Goals of the Hary and Massaro (1982) Study}

Hary and Massaro (1982) challenged the relevance of the identification-discrimination task to the theory of categorical perception with the following demonstration. They began with a "standard" rise-time continuum which gave "continuous," rather than "categorical," results (Rosen \& Howell, 1981). When these same sounds were embedded in a "bipolar" continuum, the identification-discrimination results differed significantly from those with the standard continuum and conformed to prototypical categoricalperception results (Studdert-Kennedy et al. 1970). The identification function had a relatively sharp boundary with a corresponding peak in discrimination performance near this boundary. Thus, the same sounds gave continuous results in one situation and categorical results in another.

There are two general interpretations that might be reached given the Hary and Massaro (1982) results. First, the fundamental processes governing the perception of a sound might depend on the nature of other sounds used in the experiment. Second, the form of the identification-discrimination results cannot be taken as diagnostic of whether perception is categorical or continuous. Given the lack of evidence or theory for the first alternative and its unparsimonious nature, the second is the more reasonable conclusion. It is all the more reasonable because, at the theoretical level, continuous models of perception can predict categorical results. The experiment represents a demonstration of the nondiagnosticity of the relationship between identification and discrimination performance.

Although many researchers may persist in looking at the relationship between identification and discrimination, we believe that the current use of this paradigm is without merit. The primary reason is that it is not being used to distinguish among alternative theories of performance. Furthermore, one should not necessarily expect a relationship between identification and discrimination, even though theories of both categorical and continuous perception can be formulated to predict such a relationship, and a relationship is often found. By definition, categorization behavior requires that the perceiver identify discriminable events as members of the same category. Thus, the relative probabilities of identification can not be taken as an index of relative discriminability, and discrimination will usually be better than that predicted from identification, as is usually found (Macmillan, 1983; Miller, 1956).

Hary and Massaro (1982) discussed two possible explanations of the observed differences between the standard and bipolar continua. The bipolar continuum might have contained a perceptual discontinuity at the change from negative to positive rise times. A perceived change in the direction of the rise time would be much more discriminable than the quantitative changes within either the negative or the positive rise times (Massaro \& Oden, 1980). This perceptual attribute would give good discrimination between instances on opposite poles of the perceptual discontinuity in the bipolar continuum.

The second explanation had to do with category labels mediating discrimination in the same-different task, but not as assumed by the dual coding model (Fujisaki \& Kawashima, Note 1). According to their model, the listener first compares the items at a categorical level. If a decision cannot be made at this level, the items are compared at an auditory level. It seems more reasonable to assume that the auditory distinction between the sounds is primary and the category labels play a role only if the subject fails to discriminate the sounds at an auditory level. If two sounds are not discriminated at the auditory level, the subject may still respond different when the sounds are given different category labels. On the other hand, if the sounds are not discriminated at the auditory level and are given the same category label, subjects would tend to respond same. Hence, the same two sounds would be discriminated differently as a function of how the sounds were categorized. Differences between the discrimination results for the two continua might be somewhat due to corresponding differences in categorization.

\section{Macmillan's Psychophysical Account}

Macmillan proposes three well-founded psychophysical principles to account for the Hary and Massaro (1982) identification/discrimination results: Weber's law, range effects, and anchor effects. Weber's law predicts equal discriminability for logarithmically spaced stimuli, discriminability decreases with increases in the range of stimuli used in the task, and discriminability improves near anchors (easily memorized stimuli). Given these three mechanisms, Macmillan generated predicted results for the discrimination results for the standard and bipolar continua. He concluded that the predictions "provide a good qualitative description of the data" (p. 496).

We are not impressed with this psychophysical account of the results. The assumption that the 0 -msec stimulus is an anchor in this task is post hoc and arbitrary. If anything, the performance indicates that the 15- and 45-msec stimuli are the best discriminated 
in the task. This would put the hypothetical anchor at a positive rise time rather than at the zero rise time. In addition, there are three points on the discrimination curves that occur in both the standard and bipolar continua, and in all cases the psychophysical theory makes the wrong prediction concerning the direction of the differences between each pair of points.

Macmillan's (1983) selective test of the continuous psychophysical account represents the same confirmation bias of previous advocates of categorical perception. To evaluate the quantitative description given by the psychophysical model and to contrast it with the description given by categorical perception, we computed the root mean squared deviation (RMSD) between predicted and observed percentagecorrect values. The $\mathrm{d}^{\prime}$ values given in Figure $2 \mathrm{c}$ of Macmillan's paper were translated into hit and falsealarm probabilities to match the observed hit and false-alarm probabilities as much as possible. The predicted percentage-correct values were computed from these probabilities. Macmillan's psychophysical account gave RMSD values of .060 and .066 for the bipolar and standard continua, respectively. The categorical model gave RMSD values of .059 and .092 for the bipolar and standard continua, respectively. Given that Macmillan's psychophysical account is not significantly better than the account given by the theory of categorical perception, there is no basis to accept a continuous over a categorical description of the results. The identification/discrimination task cannot discriminate between these two models.

Contrary to the implication of Macmillan's (1983) discussion of the same-different task, the differencing model that he proposes is not necessarily the most accurate description of a subject's performance in the task. As in so many cases of decision making, the most optimal strategy seldom captures psychological reality (Lopes, 1981). The decision process that is used is an empirical question, and there are no definitive data for pluck/bow discrimination. Creelman and Macmillan (1979) observed that the differencing model was a reasonable description of auditory frequency discrimination, but not of phase discrimination. Performance in the same-different task was about two times greater than the predictions of the differencing model. As suggested by Creelman and Macmillan (1979), subjects might regard the pair of signals as a single event and base their decision on the absolute change between the two signals. This strategy, among others, would lead to two normal equal-variance distributions for "same" and "different" trials, making it appropriate to analyze the samedifferent task by subtracting z scores. Even though we cannot agree on the appropriate method of calculating $\mathrm{d}^{\prime}$, it is noteworthy that the two different methods lead to essentially identical conclusions.

\section{Conclusion}

The original interpretation of the results by Hary and Massaro is still valid. The standard continuum gave results that would be interpreted as reflecting continuous perception, whereas the bipolar continuum gave results that would be interpreted as reflecting categorical perception. Given that some of the same stimuli were present in both conditions, the categorical results cannot mean that the stimuli could not be perceived continuously. The standard continuum with these stimuli gave results that normally support the idea that the stimuli are perceived continuously rather than categorically. The results dictate that we must reject the identification-discrimination paradigm as an index of whether perception is categorical or continuous. Other techniques, such as those used by Massaro and Cohen (1983), must be used to assess the nature of the process.

In closing, we can ask whether our main conclusion is paradoxical, as suggested by Macmillan (1983). The conclusion, also stated in the title of the Hary and Massaro (1982) paper, is that categorical results in the identification-discrimination task do not necessarily imply that perception is categorical. Our conclusion is paradoxical only if the theoretical explanation is equated with the empirical result, an unreasonable scientific strategy. We hope that the important differences between results and theory are respected, and conclude that the identification/ discrimination paradigm cannot distinguish between categorical and continuous theories of perception.

\section{REFERENCE NOTE}

1. Fujisaki, H., \& Kawashima, T. Some experiments on speech perception and a model for the perceptual mechanisms (Annual Report, Engineering Research Institute, Vol. 29, pp. 206-214). Tokyo: University of Tokyo, Faculty of Engineering, 1970.

\section{REFERENCES}

Creelman, C. D., \& Macmillan, N. A. Auditory phase and frequency discrimination: A comparison of nine procedures. Journal of Experimental Psychology: Human Perception and Performance, 1979, 5, 146-156.

Cutring, J. E., \& Rosner, B. S. Categories and boundaries in speech and music. Perception \& Psychophysics, 1974, 16, 564-570.

Durlach, N. I., \& Braida, L. D. Intensity perception. I. Preliminary theory of intensity resolution. Journal of the Acoustical Society of America, 1969, 46, 372-383.

Hary, J. M., \& MAssaro, D. W. Categorical results do not imply categorical perception. Perception \& Psychophysics, 1982, 32, 409-418.

Healy, A. F., \& RePp, B. H. Context independence and phonetic mediation in categorical perception. Journal of Experimental Psychology: Human Perception and Performance, 1982, 8, 68-80.

Liberman, A. M., Harris, K. S., Hoffman, H. S., \& Griftith, B. C. The discrimination of speech sounds within and across phoneme boundaries. Journal of Experimental Psychology, 1957, 54, 358-368.

Lopes, L. Decision making in the short run. Journal of Experi- 
mental Psychology: Human Learning and Memory, 1981, 7, 377-385.

Macmillan, N. A. A psychophysical interpretation of a "categorical perception" experiment by Hary and Massaro. Perception \& Psychophysics, 1983, 34, 494-498.

Macmillan, N. A., Kaplan, H. L., \& Creelman, C. D. The psychophysics of categorical perception. Psychological Review, $1977,84,452-471$.

Massaro, D. W., \& Cohen, M. M. Categorical or continuous speech perception: A new test. Speech Communication, 1983, 2, 15-35.

Massaro, D. W., \& Oden, G. C. Speech perception: A framework for research and theory. In N. J. Lass (Ed.), Speech and language: Advances in basic research and practice (Vol. 3). New York: Academic Press, 1980.
Millen, G. A. The magical number seven, plus or minus two: Some limits on our capacity for processing information. Psychological Review, 1956, 63, 81-97.

POPPER, K. The logic of scientific discovery. New York: Basic Books, 1959.

Rosen, S. M., \& Howell, P. Plucks and bows are not categorically perceived. Perception \& Psychophysics, 1981, 30, 156-168.

Studdert-Kennedy, M., Liberman, A. M., Harris, K. S., \& Coopen, F. S. Motor theory of speech perception: A reply to Lane's critical review. Psychological Review, 1970, 77, 234-249.

(Manuscript received August 23, 1983; accepted for publication August 23, 1983.) 